\title{
UNITS AND ONE-SIDED UNITS IN REGULAR RINGS
}

BY

\section{GERTRUDE EHRLICH}

\begin{abstract}
A ring $R$ is unit regular if for every $a \in R$, there is a unit $x \in R$ such that $a x a=a$, and one-sided unit regular if for every $a \in R$, there is a right or left invertible element $x \in R$ such that $a x a=a$. In this paper, unit regularity and one-sided unit regularity are characterized within the lattice of principal right ideals of a regular ring $R$ (Theorem 3). If $M$ is an $A$-module and $R=$ End $_{A} M$ is a regular ring, then $R$ is unit regular if and only if complements of isomorphic summands of $M$ are isomorphic, and $R$ is one-sided unit regular if and only if complements of isomorphic summands of $M$ are comparable with respect to the relation "is isomorphic to a submodule of" (Theorem 2). A class of modules is given for whose endomorphism rings it is the case that regularity in conjunction with von Neumann finiteness is equivalent to unit regularity. This class includes all abelian torsion groups and all nonreduced abelian groups with regular endomorphism rings.
\end{abstract}

In [1], a ring $R$ with identity was defined to be unit regular if for every $a \in R$ there is a unit $x \in R$ such that $a x a=a$. The class of all unit regular rings includes [1] all semisimple Artinian rings, all continuous von Neumann rings [7], all strongly regular rings (in particular, all commutative regular rings [3]). Using the characterization (cf. [6, p. 117]) of regular group rings as the group rings of locally finite groups, it is easy to show that all regular group rings are unit regular. Unit regular rings are von Neumann finite [4, Proposition 1] and are elementary divisor rings [4, Theorem 3]. Every element of a unit regular ring in which 2 is a unit is equal to the sum of two units [1, Theorem 6]. An example of a regular ring which is not unit regular is the endomorphism ring of an infinite dimensional vector space [1].

In this paper, we define one-sided unit regularity and characterize both unit regularity and one-sided unit regularity within the lattice of principal right ideals of the ring (Theorem 3). For an $A$-module $M$ with regular endomorphism ring $R$ we prove that $R$ is unit regular if and only if any two isomorphic complemented

Presented to the Society, March 19, 1974; received by the editors April 4, 1974 and, in revised form, September 9, 1974.

AMS (MOS) subject classifications (1970). Primary 16A30; Secondary 16A42, 16A48, 16A64, 20K30.

Key words and phrases. Regular ring, unit regular ring, one-sided unit regular ring, von Neumann finite ring, endomorphism ring, lattice of complemented submodules, cancellation property, reduced abelian torsion group, nonreduced abelian group. 
submodules of $M$ have isomorphic complements, and that $R$ is one-sided unit regular if and only if any two isomorphic complemented submodules of $M$ have comparable complements (Theorem 2). (We call two $A$-modules comparable if one of them is isomorphic to a submodule of the other.)

In response to the question posed in [4] as to whether every von Neumann finite regular ring with identity is unit regular, we produce (Theorem 4 ) a class of $A$-modules, $M$, whose endomorphism ring $R=\mathrm{End}_{A} M$ is regular and von Neumann finite if and only if it is unit regular. (This class of modules includes, in particular, all nonreduced abelian groups and all reduced abelian torsion groups with regular endomorphism rings.) Within this class, we characterize those modules whose endomorphism rings, if regular, are one-sided unit regular. We prove (Corollary, Theorem 1) that the endomorphism ring of any vector space is one-sided unit regular and give examples of regular rings which are not one-sided unit regular. Finally (Theorem 5), we prove that a one-sided unit regular ring in which 2 is invertible is generated either by its left invertible elements or by its right invertible elements.

Notations. 1. If $R$ is a ring, $a \in R$, we write $(R a)^{r}$ for the right annihilator of the principal left ideal generated by $a$, and we write $(a R)^{l}$ for the left annihilator of the principal right ideal generated by $a$.

2. If $X, Y$ are submodules of an $A$-module $M$, we write $X \widetilde{\subset} Y$ to indicate that $X$ is isomorphic to a submodule of $Y$. (We say that two submodules $X$ and $Y$ are comparable if either $X \widetilde{\subset} Y$ or $Y \widetilde{\subset} X$ holds.)

3. If $R$ is a ring, we write $R_{r}$ for the right $R$-module $R$.

REMARKs. 1. If $M$ is an $A$-module, then $R=\operatorname{End}_{A} M$ is regular if and only if for each $a \in R$, both $\operatorname{Im} a$ and $\operatorname{Ker} a$ are direct summands of $M$ [9, Corollary 3.2] .

2. If $M$ is an $A$-module such that $R=\operatorname{End}_{A} M$ is regular and $x \in R$, then

(a) $x$ is a unit in $R$ if and only if it is an automorphism of $M$;

(b) $x$ is right invertible in $R$ if and only if it is an epimorphism of $M$;

(c) $x$ is left invertible in $R$ if and only if it is a monomorphism of $M$.

(NotE. The regularity of $R$ is needed to obtain the backward implications of (b) and (c).)

3. Let $M$ be an $A$-module such that $R=$ End $_{A} M$ is regular. Then $R$ is von Neumann finite if and only if $M$ is not isomorphic to a proper submodule of itself. (This follows immediately from Remark 2.)

4. If $R$ is a regular ring with identity, then the principal right (left) ideals of $R$ form a complemented modular, hence relatively complemented lattice $[6, \mathrm{p} .5]$, [8, Theorem 2]. The maps $a R \rightarrow(a R)^{l}$ and $R a \rightarrow(R a)^{r}$ are inverse anti-isomorphisms between the lattice of all principal right ideals and the lattice of all principal left ideals of $R \quad$ [8, Theorem 1].

Definition. Let $R$ be a ring with identity. If $a \in R$, then 
(i) $a$ is unit regular if there is a unit $x \in R$ such that axa $=a$;

(ii) $a$ is right unit regular if there is a right invertible element $x \in R$ such that $\operatorname{axa}=a$;

(iii) $a$ is left unit regular if there is a left invertible element $x \in R$ such that $a x a=a$.

$R$ is unit regular if every element of $R$ is unit regular [1].

$R$ is one-sided unit regular if every element of $R$ is either right or left unit regular.

The following lemma establishes a basic correspondence between the complemented submodules of a right $A$-module $M$ with regular endomorphism ring $R$ and the principal right ideals of $R$.

Lemma 1. Let $A$ be a ring with identity and let $M$ be a right $A$-module such that $R=\operatorname{End}_{A} M$ is a regular ring. Let $L_{P}(R)$ be the lattice of all principal right ideals of $R$ and let $L_{C}(M)$ be the set of all complemented submodules of $M$. Then

(1) $L_{C}(M)$ is a sublattice of the lattice of all submodules of $M$;

(2) $\varphi: a R \mapsto a M(a \in R)$ is an isomorphism of the lattice $L_{P}(R)$ onto the lattice $L_{C}(M)$.

(3) If $a \in R$, then $\operatorname{Ker} a=\varphi(R a)^{r}$.

Proof. We prove first that $\varphi$ is a bijection of $L_{P}(R)$ onto $L_{C}(M)$. If $a$, $b \in R$ and $a R=b R$, then clearly $a M=b M$. If $H$ is a principal right ideal of $R$, then $H=e R$ for some idempotent $e \in R$. Hence $\varphi(H)=e M$ and $(1-e) M$ is a complement of $e M$ in $M$, whence $\varphi(H) \in L_{C}(M)$. If $e, f \in R$ are idempotents such that $e M=f M$, then for each $\alpha \in M, e \alpha=f e \alpha$ and $f \alpha=e f \alpha$, hence $e=$ $f e, f=e f$, and so $e R=f R$. Thus, $\varphi$ is an injective map of $L_{P}(R)$ into $L_{C}(M)$. If $L \in L_{C}(M)$, then any projection map of $M$ onto $L$ is an idempotent $e \in R$ such that $L=e M=\varphi(e R)$. It follows that $\varphi: L_{P}(R) \rightarrow L_{C}(M)$ is a bijection. If $e, f \in R$ are idempotents, then $e R \subset f R$ if and only if $e M \subset f M$. It remains to be shown that $L_{C}(M)$ is closed under + and $\cap$. Let $e, f \in R$ be idempotents and let $g \in R$ be an idempotent such that $g R=e R+f R$. Then $g e=e, g f=f$, and so $e M+f M \subset g M$. Since $g=e s+f t$ for some $s, t \in R, g M \subset e M+f M$. But then $e M+f M=g M \in L_{C}(M)$. Finally, let $e, f \in R$ be idempotents and let $g \in R$ be an idempotent such that $e R \cap f R=g R$. Then $g=e g=f g$, whence $g M \subset e M \cap f M$. Now,

$$
\begin{aligned}
g R & =e R \cap f R=(R(1-e))^{r} \cap(R(1-f))^{r} \\
& =(R(1-e)+R(1-f))^{r}=(R(1-g))^{r} .
\end{aligned}
$$

Hence $R(1-g)=R(1-e)+R(1-f)$, and $1-g=x(1-e)+y(1-f)$ for some $x, y \in R$. But then, for $\alpha \in e M \cap f M$, we have $\alpha=e \alpha=f \alpha$, and $(1-g) \alpha$ 
$=x(1-e) e \alpha+y(1-f) f \alpha=0$. Hence $e M \cap f M \subset \operatorname{Ker}(1-g)$. It is trivial to check that $\operatorname{Ker}(1-g)=g M$. We conclude that $e M \cap f M=g M \in L_{C}(M)$.

Thus, $L_{C}(M)$ is a sublattice of the lattice of all submodules of $M$ and $\varphi: L_{P}(R)$ $\rightarrow L_{C}(M)$ is a lattice isomorphism. Finally, (3) follows from the fact that, if $a \in R$ and $f \in R$ is an idempotent such that $R a=R f$, then $\operatorname{Ker} a=\operatorname{Ker} f=$ $(1-f) M=\varphi(1-f) R=\varphi(R a)^{r}$.

(NoTE. We make repeated use of the fact that $L_{C}(M)$, like $L_{P}(R)$, is a relatively complemented lattice.)

Theorem 1. Let $A$ be a ring with identity and let $M$ be a right $A$-module such that $R=\operatorname{End}_{A} M$ is a regular ring. Let $a \in R$.

I. The following statements are equivalent:

(1) $a$ is unit regular,

(2) there is an automorphism $u: M \rightarrow M$ such that $\operatorname{Im} a \oplus u \operatorname{Ker} a=M$,

(3) $\operatorname{Ker} a \cong$ Coker $a$.

II. The following statements are equivalent:

(1) $a$ is right unit regular,

(2) there is a monomorphism $u: M \rightarrow M$ such that $\operatorname{Im} a \cap u \operatorname{Ker} a=0$,

(3) Ker $a \widetilde{\subset}$ Coker $a$.

III. The following statements are equivalent:

(1) $a$ is left unit regular,

(2) there is an epimorphism $u: M \rightarrow M$ such that $\operatorname{Im} a+u \operatorname{Ker} a=M$,

(3) there is an epimorphism $u: M \rightarrow M$ such that $\operatorname{Im} a \oplus u \operatorname{Ker} a=M$,

(4) Coker $a \widetilde{\subset} \operatorname{Ker} a$.

(Recall that Coker $a=M / \operatorname{Im} a$, isomorphic to any complement of $\operatorname{Im} a$ in $M$.)

Proof. I. (1) $\Rightarrow(2)$. Suppose $a$ is unit regular. Let $x \in R$ be a unit such that $a x a=a$ and let $u=x^{-1}$. Then $f=x a$ is an idempotent such that $R a=R f$. Hence Ker $a=(1-f) M$ and we have $M=u M=u(f M \oplus(1-f) M)=u f M \oplus$ $u(1-f) M=\operatorname{Im} a \oplus u \operatorname{Ker} a$.

(2) $\Rightarrow$ (3). If $u: M \rightarrow M$ is an automorphism such that $M=\operatorname{Im} a \oplus u \operatorname{Ker} a$, then Coker $a=M / \operatorname{Im} a \cong u \operatorname{Ker} a \cong \operatorname{Ker} a$.

(3) $\Rightarrow$ (1). Suppose Ker $a \widetilde{\subset}$ Coker $a$. Let $T$ be a complement of Ker $a$ in $M$, and let $S$ be a complement of $\operatorname{Im} a$ in $M$. Then $\left.a\right|_{T}$ is injective. Let $\beta: \operatorname{Im} a$ $\rightarrow T$ be the isomorphism which is inverse to $\left.a\right|_{T}$ and let $\mu: S \rightarrow \operatorname{Ker} a$ be an isomorphism. Then the map $x: M \rightarrow M$ defined by $\left.x\right|_{\operatorname{Im} a}=\beta$ and $\left.x\right|_{S}=\mu$ is an automorphism of $M$. If $\tau \in T$, then $a x a \tau=a \beta a \tau=a \tau$, and if $\kappa \in \operatorname{Ker} a$, then $\operatorname{axak}=0=a \kappa$. Hence $a x a=a$, with $x$ a unit in $R$.

II. (1) $\Rightarrow(2)$. Suppose $a$ is right unit regular. Let $x \in R$ be a right invertible element such that $a x a=a$ and let $u \in R$ be a right inverse of $x$. Then $u$ : $M \rightarrow M$ is a monomorphism and $\operatorname{Im} a \cap u \operatorname{Ker} a=0$. For: If $a \xi=u k \quad(\xi \in M$, 
$\kappa \in \operatorname{Ker} a)$, then $a \xi=a x a \xi=a x u \kappa=a \kappa=0$.

(2) $\Rightarrow$ (3). Suppose there is a monomorphism $u: M \rightarrow M$ such that $\operatorname{Im} a \cap$ $u \operatorname{Ker} a=0$. Let $Q$ be a submodule of $M$ such that $M=(\operatorname{Im} a \oplus u \operatorname{Ker} a) \oplus Q$. Then $u \operatorname{Ker} a \oplus Q$ is a complement of $\operatorname{Im} a$ in $M$ and we have Ker $a \cong u$ Ker $a \widetilde{C}$ $u \operatorname{Ker} a \oplus Q \cong$ Coker $a$.

(3) $\Rightarrow(1)$. Suppose Ker $a \widetilde{\subset}$ Coker $a$. Let $\alpha$ : Ker $a \rightarrow$ Coker $a$ be a monomorphism. Define $y: M \rightarrow M$ by: $\left.y\right|_{T}=\left.a\right|_{T}$ and $\left.y\right|_{\text {Ker } a}=\alpha(T$ a complement of $\operatorname{Ker} a$ in $M$ ). Then $y$ is a monomorphism, hence left invertible in $R$. Let $x \in$ $R$ be a left inverse of $y$. Then for $\tau \in T$, axa $=a x y \tau=a \tau$, and for $\kappa \in \operatorname{Ker} a$, $\operatorname{axak}=0=a \kappa$. Thus $a x a=a$, with $x$ right invertible.

III. (1) $\Rightarrow(2)$. Suppose $a$ is left unit regular. Let $x \in R$ be a left invertible element such that $a x a=a$ and let $u \in R$ be a left inverse of $x$. Then $u: M \rightarrow M$ is an epimorphism and, for $f=x a$, we have $R a=R f$ and $M=f M \oplus(1-f) M=$ $=u f M+u(1-f) M=\operatorname{Im} a+u \operatorname{Ker} a$.

(2) $\Rightarrow$ (3). Suppose $M=\operatorname{Im} a+u \operatorname{Ker} a$, where $u: M \rightarrow M$ is an epimorphism. Since $u \operatorname{Ker} a=\varphi\left(u(R a)^{r}\right), \operatorname{Im} a \cap u \operatorname{Ker} a$ is a complemented submodule of $M$, hence of $u \operatorname{Ker} a$. Let $Q$ be a submodule of $M$ such that $(\operatorname{Im} a \cap u \operatorname{Ker} a$ ) $\oplus Q=u \operatorname{Ker} a$. Then $Q$ is a homomorphic image of $u \operatorname{Ker} a$, and $M=\operatorname{Im} a \oplus Q$. Hence there is an epimorphism $\bar{u}: M \rightarrow M$ such that $\left.\bar{u}\right|_{T}=\left.a\right|_{T} \quad(T$ a complement of $\operatorname{Ker} a$ in $M$ ) and $\left.\bar{u}\right|_{\operatorname{Ker} a}$ is an epimorphism mapping $\operatorname{Ker} a$ onto $Q$. Thus, $M=\operatorname{Im} a \oplus \bar{u} \operatorname{Ker} a$, with $\bar{u}: M \rightarrow M$ an epimorphism.

(3) $\Rightarrow$ (4). Suppose $u: M \rightarrow M$ is an epimorphism such that $\operatorname{Im} a \oplus u \operatorname{Ker} a$ $=M$. Let $L$ be a submodule of $M$ such that $(\operatorname{Ker} a \cap \operatorname{Ker} u) \oplus L=\operatorname{Ker} a$. Then Coker $a \cong u$ Ker $a \cong \operatorname{Ker} a /(\operatorname{Ker} a \cap \operatorname{Ker} u) \cong L \subset \operatorname{Ker} a$. whence Coker $a \widetilde{C} \operatorname{Ker} a$.

(4) $\Rightarrow(1)$. Suppose Coker $a \widetilde{C} \operatorname{Ker} a$. Let $T$ be a complement of $\operatorname{Ker} a$ in $M$ and let $S$ be a complement of $\operatorname{Im} a$ in $M$. Let $\beta: \operatorname{Im} a \rightarrow T$ be the isomorphism which is inverse to $\left.a\right|_{T}$, and let $\alpha: S \rightarrow \operatorname{Ker} a$ be a monomorphism. Then there is a monomorphism $x: M \rightarrow M$ such that $\left.x\right|_{\operatorname{Im} a}=\beta$ and $\left.x\right|_{S}=\alpha$. If $\tau \in T$, then $\operatorname{axa} \tau=a \beta a \tau=a \tau$, and if $\kappa \in \operatorname{Ker} a$, then $a x a \kappa=0=a \kappa$. Thus, axa $a$, with $x$ left invertible.

COROLlaRY. Let $M$ be a vector space over a division ring $D$ and let $R=$ End $_{D} M$.

(1) If $a \in R$, then $a$ is unit regular if and only if $\operatorname{dim} \operatorname{Ker} a=\operatorname{dim} \operatorname{Coker} a$; $a$ is right unit regular if and only if $\operatorname{dim} \operatorname{Ker} a \leqslant \operatorname{dim} \operatorname{Coker} a ; a$ is left unit regular if and only if $\operatorname{dim}$ Coker $a \leqslant \operatorname{dim} \operatorname{Ker} a$.

(2) $R$ is one-sided unit regular.

(3) $R$ is unit regular if and only if $M$ is finite dimensional.

Proof. (1) and (2) are immediate consequences of Theorem 1. (3) was proved in [1]. 
Theorem 2 (C Ancellation Property). Let $A$ be a ring with identity, $M$ a right $A$-module such that $R=\mathrm{End}_{A} M$ is a regular ring.

(1) $R$ is unit regular if and only if, for submodules $P_{1}, P_{2}, Q_{1}, Q_{2}$ such that $M=P_{1} \oplus Q_{1}=P_{2} \oplus Q_{2}, P_{1} \cong P_{2}$ implies $Q_{1} \cong Q_{2}$ (i.e., complements of isomorphic submodules are isomorphic).

(2) $R$ is one-sided unit regular if and only if, for submodules $P_{1}, P_{2}, Q_{1}$, $Q_{2}$ such that $M=P_{1} \oplus Q_{1}=P_{2} \oplus Q_{2}, P_{1} \cong P_{2}$ implies either $Q_{1} \widetilde{\subset} Q_{2}$ or $Q_{2} \widetilde{C}$ $Q_{1}$ (i.e., complements of isomorphic submodules are comparable.)

Proof. It suffices to note that, if $M=P_{1} \oplus Q_{1}=P_{2} \oplus Q_{2}$, with $P_{1} \cong$ $P_{2}$, then there is an endomorphism $a: M \rightarrow M$ such that $P_{2}=\operatorname{Im} a$ and $Q_{1}=$ Ker $a$. (In fact, any isomorphism of $P_{1}$ onto $P_{2}$ can be extended to such an endomorphism.) Thus, Theorem 1 applies and (1) and (2) follow immediately.

THEOREM 3. I. Let $R$ be a regular ring with identity and let $a \in R$. Then

(1) $a$ is unit regular if and only if

(i) there is a unit $u \in R$ such that $a R \oplus u(R a)^{r}=R$;

(2) $a$ is right unit regular if and only if

(ii) there is a left invertible element $u \in R$ such that $a R \cap u(R a)^{r}=0$;

(3) $a$ is left unit regular if and only if

(iii) there is a right invertible element $u \in R$ such that $a R+u(R a)^{r}=R$.

II. A ring $R$ with identity is unit regular if and only if (i) holds for each $a \in R$.

III. A regular ring $R$ with identity is one-sided unit regular if and only if either (ii) or (iii) holds for each $a \in R$.

Proof. Apply Theorem 1 to the right $R$-module $R_{r}$, noting that (a) $R$ is isomorphic to $\operatorname{End}_{R}\left(R_{r}\right)$ under the regular representation which associates with each $a \in R$ the left multiplication, $a_{L}$, determined by $a$; (b) for $a \in R, a R=$ $\operatorname{Im} a_{L}$ and $(R a)^{r}=\operatorname{Ker} a_{L}$; (c) if $u \in R$, then $u$ is a unit if and only if $u_{L}$ is an automorphism, $u$ is right invertible if and only if $u_{L}$ is an epimorphism, and $u$ is left invertible if and only if $u_{L}$ is a monomorphism. (The last two assertions are based on the regularity of $R$.)

Note that regularity of $R$ need not be assumed in II since (i) implies that every principal right ideal of $R$ is generated by an idempotent. Note also that in I(3), + may be replaced by $\oplus$.

(Analogous characterizations may be given within the lattice of principal left ideals of $R$.)

To construct examples of regular rings which are not one-sided unit regular, we make use of the fact that, if $R$ is a direct sum (product) of rings $R_{i}$, then $a \in$ $R$ is regular, unit regular, right or left unit regular if and only if each component 
$a_{i}$ of $a \quad\left(a_{i} \in R_{i}\right)$ has the corresponding property. In particular, if $R_{1}$ and $R_{2}$ are regular rings which are not von Neumann finite and $R=R_{1} \oplus R_{2}$, we may take $a_{1} \in R_{1}$ right but not left invertible and $a_{2} \in R_{2}$ left but not right invertible. Then $a_{1}$ is left but not right unit regular, $a_{2}$ is right but not left unit regular, and so $\left(a_{1}, a_{2}\right) \in R$ is neither right nor left unit regular, while $R$ is clearly regular. Specifically, we may take $R=$ End $G$, where $G$ is the abelian group given by: $G=H \oplus K$, with $H$ the direct sum of infinitely many isomorphic copies of $Z_{p}$, $K$ the direct sum of infinitely many isomorphic copies of $Z_{q}$ ( $p, q$ distinct primes). Since all subgroups of $H$ and $K$ are direct summands, End $H$, End $K$ and hence End $G$ are regular (see Remark 1, p. ). Since $H$ and $K$ are fully invariant subgroups of $G$, End $G=$ End $H+$ End $K$. By Remark 4, p. , neither End $H$ nor End $K$ is von Neumann finite. Hence End $G$ is not one-sided unit regular.

Lemma 2. Let $A$ be a ring with identity, $M$ a right $A$-module such that $R=$ End $_{A} M$ is regular. Suppose $M$ is expressible as a direct sum of isomorphic indecomposable submodules of $M$. Then

(1) $R$ is one-sided unit regular;

(2) $R$ is unit regular if and only if $M$ is a direct sum of finitely many isomorphic indecomposable submodules.

Proof. (1) Let $Y$ be an indecomposable $A$-module and let $\left\{Y_{j}\right\}_{j \in J}$ be a family of submodules of $M$ such that $M=\bigoplus_{j \in J} Y_{j}$, with each $Y_{j}$ isomorphic to $Y$. Suppose that $R=\operatorname{End}_{A} M$ is regular. To prove that $R$ is one-sided unit regular, it suffices to prove that any two complemented submodules of $M$ are comparable.

Let $N$ be a complemented submodule of $M$. By Lemma 1 , there is an endomorphism $a \in R$ such that $N=\operatorname{Im} a=\Sigma_{j \in J} a Y_{j}$. For each $j \in J,\left.\operatorname{Ker} a\right|_{Y_{j}}=$ $\operatorname{Ker} a \cap Y_{j}$ is a direct summand of $M$, hence of $Y_{j}$. It follows that $\left.\operatorname{Ker} a\right|_{Y_{j}}=0$ or $\left.\operatorname{Ker} a\right|_{Y_{j}}=Y_{j}$, whence either $a Y_{j} \cong Y_{j} \cong Y$, or $a Y_{j}=0$. Thus, there is a subset $H$ of the index set $J$ such that $N=\operatorname{Im} a=\Sigma_{j \in H} a Y_{j}$, where $a Y_{j} \neq 0$ for $j \in H$. We may choose $H$ small enough to ensure that, for each $h \in H, a Y_{h} \not \subset$ $\Sigma_{j \in H-\{h\}} a Y_{j}$. But then $N=\bigoplus_{j \in H} a Y_{j}$. (For: Suppose that, for some $h \in$ $H, Q_{h}=a Y_{h} \cap \Sigma_{j \in H-\{h\}} a Y_{j} \neq 0$. Since there are endomorphisms of $M$ with image $a Y_{h}$ and $\Sigma_{j \in H-\{h\}} a Y_{j}$, respectively, we conclude that $Q_{h}$ is a direct summand of $M$ and therefore of $a Y_{h}$. But then $Q_{h}=a Y_{h}$, and $a Y_{h} \subset$ $\Sigma_{j \in H-\{h\}} a Y_{j}$, contrary to the choice of $H$.) Thus, $N$ is a direct sum of isomorphic copies of $Y$. Now, if $N_{1}$ and $N_{2}$ are complemented submodules of $M$ such that $N_{1}=\bigoplus_{j \in H_{1}} Y_{j}, N_{2}=\bigoplus_{j \in H_{2}} Y_{j}\left(H_{1} \subset J, H_{2} \subset J\right)$, then either Card $H_{1} \leqslant$ Card $H_{2}$, or Card $H_{2} \leqslant \operatorname{Card} H_{1}$, whence either $N_{1} \widetilde{\subset} N_{2}$ or $N_{2} \widetilde{\subset} N_{1}$. 
(2) If $R$ is unit regular, then $R$ is von Neumann finite, hence $M$ is not isomorphic to a proper submodule of itself. But then the index set $J$ is finite. Conversely, suppose $M=\bigoplus_{j=1}^{n} Y_{j}$ ( $n$ a positive integer) where the $Y_{j}$ are isomorphic indecomposable submodules of $M$. By Lemma 1 , it follows that the right $R$-module $R$ is equal to the direct sum of the principal right ideals $\varphi^{-1}\left(Y_{j}\right)(j=1, \ldots, n)$. Since the indecomposable submodules $Y_{j}$ are minimal in the lattice of complemented submodules of $M$, the $\varphi^{-1}\left(Y_{j}\right)$ are minimal principal, hence minimal, right ideals in $R$. It follows that $R$ is semisimple Artinian, hence unit regular.

THEOREM 4. Let $A$ be a ring with identity and let $M$ be a right A-module such that $M=\bigoplus_{i \in I} M_{i}$, where each $M_{i}$ is a fully invariant submodule, equal to a direct sum of isomorphic indecomposable submodules. Suppose that $R=$ End $_{A} M$ is regular. For each $i \in I$, let $R_{i}=\operatorname{End}_{A} M_{i}$. Then

(1) $R$ is unit regular if and only if it is von Neumann finite;

(2) $R$ is one-sided unit regular if and only if $R_{i}$ is von Neumann finite for all but (possibly) a single index $i \in I$.

Proof. First note that $R=\bigoplus_{i \in I} R_{i}$ (cf. [2, p. 220]), and that each $R_{i}$ is regular.

(1) We know that unit regularity implies von Neumann finiteness. Suppose $R$ is von Neumann finite. Then $M$ is not isomorphic to a proper submodule of itself. But then each $M_{i}$ is the direct sum of finitely many isomorphic indecomposable summands whence, by Lemma $2, R_{i}$ is unit regular for each $i \in I$. It follows that $R$ is unit regular.

(2) Suppose $R$ is one-sided unit regular. If $i_{1}, i_{2} \in I$ are two distinct indices such that $R_{i_{1}}$ and $R_{i_{2}}$ both fail to be von Neumann finite, we may proceed as in the examples preceding Lemma 2 to construct an endomorphism of $M_{i_{1}} \oplus$ $M_{i_{2}}$ which is not one-sided unit regular, and then extend this endomorphism to an endomorphism of $M$ which is not one-sided unit regular. Thus, at most one of the $R_{i}$ can fail to be von Neumann finite.

Conversely, suppose that all but possibly a single one of the $R_{i}$ are von Neumann finite. If all of the $R_{i}$ are von Neumann finite, then $R$ is unit regular, by part (i). If there is an index $h \in I$ such that $R_{h}$ is not von Neumann finite while for all $i \neq h \quad(i \in I), R_{i}$ is von Neumann finite, then Lemma 2 implies that $R_{h}$ is one-sided unit regular, while for $i \neq h$ ( $\left.i \in I\right), R_{i}$ is unit regular. From this, it follows easily that $R$ is one-sided unit regular.

CoRollary. Let $G$ be an abelian group such that End $G$ is regular. If $G$ is a nonreduced group, or a reduced torsion group, then $R$ is unit regular if and only if it is von Neumann finite.

Proof. A reduced abelian torsion group has a regular endomorphism ring 
if and only if it is a direct sum of cyclic groups of prime order; a nonreduced abelian group has a regular endomorphism ring if and only if it is a direct sum of cyclic groups of prime order and full rational groups [2, p. 243]. In either case, Theorem 4 applies.

REMARK. Despite the author's previous claims to the contrary (Notices Amer. Math. Soc. 21 (1974), A437, Abstract \#74T-A136), the general problem as to whether every von Neumann finite regular ring is unit regular remains unsolved. (1)

In view of Theorem 2 and the fact that every ring with identity may be regarded as the endomorphism ring of a module, the problem reduces to the following: does there exist, for some ring $A$ with identity, an $A$-module $M$ such that

(1) the image and the kernel of every endomorphism of $M$ is a direct summand of $M$ (i.e., End ${ }_{A} M$ is regular);

(2) $M$ is not isomorphic to a proper submodule of itself (i.e., the regular ring End $_{A} M$ is von Neumann finite);

(3) $M$ has submodules $P_{1}, P_{2}, Q_{1}, Q_{2}$ such that $M=P_{1} \oplus Q_{1}=P_{2} \oplus Q_{2}$, with $P_{1} \cong P_{2}$ and $Q_{1} \neq Q_{2}$ (i.e., the regular ring End $A$ is not unit regular)?

(Since one-sided unit regularity and unit regularity are equivalent in a von Neumann finite regular ring, the condition " $Q_{1} \neq Q_{2}$ " in (3) may be replaced by “ $Q_{1}, Q_{2}$ not comparable".)

THEOREM 5. Let $R$ be a regular ring with identity such that 2 is a unit in $R$.

(1) If $R$ is unit regular, then every element of $R$ is equal to the sum of two units.

(2) If $R$ is one-sided unit regular, then every element of $R$ is equal either to the sum of two right invertible or to the sum of two left invertible elements.

(3) If $R$ is one-sided unit regular, then one of the following statements holds:

(a) every element of $R$ is equal to a sum of right invertible elements, or

(b) every element of $R$ is equal to a sum of left invertible elements.

(4) If $R$ is one-sided unit regular and $R$ has an antiautomorphism, then every element of $R$ is equal to a sum of two right invertible elements and to a sum of two left invertible elements.

Proof. (1) was proved in [1, Theorem 6]. The key to the proof is the fact that, if 2 is a unit in $R$, then every idempotent $e \in R$ is expressible as $e=$ $(2 e-1) / 2+1 / 2$, the sum of two units. From this, it follows easily that every right unit regular element is equal to the sum of two left invertible elements, and every left unit regular element is equal to the sum of two right invertbile elements. Thus, (2) holds. To prove (3), we observe that the subsets $R_{1}$ and $R_{2}$ consisting, resepctively, of the sums of right invertible, and the sums of left in-

(1) Added in proof. Since this paper was written, George Bergmann has found an example of a regular ring which is von Neumann finite but not unit regular. 
vertible elements of $R$ form subrings of $R$. But then, by (2), if $R$ is one-sided unit regular, we have $R=R_{1} \cup R_{2}$. By a well-known exercise, it follows that either $R=R_{1}$ or $R=R_{2}$. (4) is an immediate consequence of (2).

\section{REFERENCES}

1. G. Ehrlich, Unit regular rings, Portugal. Math. 27 (1968), 209-212. MR 42 \#1864.

2. L. Fuchs, Infinite abelian groups. Vol. 2, Academic Press, New York, 1973.

3. L. Gillman and M. Henriksen, Some remarks about elementary divisor rings, Trans. Amer. Math. Soc. 82 (1956), 362-365. MR 18, 9.

4. M. Henriksen, On a classs of regular rings that are elementary divisor rings, Arch. Math. (Basel) 24 (1973), 133-141.

5. I. Kaplansky, Fields and rings, The Univ. of Chicago Press, Chicago, Ill., 1969. MR 42 \#4345.

6. L. A. Skornjakov, Complemented modular lattices and regular rings, Fizmatgiz, Moscow, 1961; English transl., Oliver \& Boyd, London, 1964. MR 29 \#3404; 30 \#42.

7. J. von Neumann, Continuous rings and their arithmetics, Proc. Nat. Acad. Sci. U.S.A. (1936), 707-713.

8. - On regular rings, Proc. Nat. Acad. Sci. U.S.A. 22 (1936), 707-713.

9. R. Ware, Endomorphism rings of projective modules, Trans. Amer. Math. Soc. 155 (1971), 233-252. MR 43 \#274.

DEPARTMENT OF MATHEMATICS, UNIVERSITY OF MARYLAND, COLLEGE PARK, MARYLAND 20742 